\title{
Selected top quark mass measurements at CMS
}

\author{
Elvire Bouvier*i \\ Institut de Physique Nucléaire de Lyon \\ Domaine scientifique de la Doua \\ 4 rue Enrico Fermi \\ F-69622 Villeurbanne (France) \\ E-mail: e.bouvieracern.ch
}

Selected measurements of the top quark mass are presented, obtained from CMS data collected in proton-proton collisions at the LHC at center-of-mass energies of 7, 8, and $13 \mathrm{TeV}$. "Standard" techniques are employed in each decay channel of top quark pair events and their results are combined. The mass of the top quark is also measured using several "alternative" methods, including measurements from shapes of top quark decay distributions in single top quark and top quark pair events as well as pole mass measurements.

38th International Conference on High Energy Physics 3-10 August 2016

Chicago, USA

\footnotetext{
* Speaker.

${ }^{\dagger}$ On behalf of the CMS collaboration.
} 
The top quark mass $\left(\mathrm{m}_{\mathrm{t}}\right)$ is a free parameter of the standard model (SM) whose precise determination contributes, in particular, as a probe of the electroweak sector of the SM. Selected measurements, either "standard" or "alternative", are presented in this report. Most of them are based on the proton-proton data recorded by the CMS detector at a center-of-mass energy $(\sqrt{s})$ of $8 \mathrm{TeV}$ and corresponding to a luminosity of $19.7 \mathrm{fb}^{-1}$. The first $\mathrm{m}_{\mathrm{t}}$ measurement performed with $13 \mathrm{TeV}$ data corresponding to $2.3 \mathrm{fb}^{-1}$ is also mentioned.

\section{1. "Standard" methods}

The so-called "standard" methods exploit the full kinematic of top quark pair (t⿱tt) events. One measurement per $\bar{t} \bar{t}$ decay channel is performed. In the all jets and lepton+jets channels, a kinematic fit is performed before simultaneously measuring $\mathrm{m}_{\mathrm{t}}$ and a global jet energy scale factor (JSF), using the ideogram method (i.e. analytical likelihood templates). The JSF is assumed to be normally distributed with 1 as expected value and the jet energy calibration uncertainty (JEC) as standard deviation. In the dilepton channel, the presence of two undetected neutrinos prevents the use of the same strategy. A matrix weighting technique is combined with an analytical algorithm to solve the kinematic equations. Each event is reconstructed several times, varying the jet momenta $\left(p_{\mathrm{T}}\right)$ with a Gaussian distribution whose standard deviation is the JEC uncertainty. Applied to $8 \mathrm{TeV}$ data, these measurements result in $\mathrm{m}_{\mathrm{t}}=172.32 \pm 0.25$ (stat) \pm 0.59 (syst) $\mathrm{GeV}$, $\mathrm{m}_{\mathrm{t}}=172.35 \pm 0.16$ (stat) \pm 0.48 (syst) $\mathrm{GeV}$, and $\mathrm{m}_{\mathrm{t}}=172.82 \pm 0.19$ (stat) \pm 1.22 (syst) GeV respectively [1]. They all rely on a simulation-based calibration, so does the nature of the measured mass.

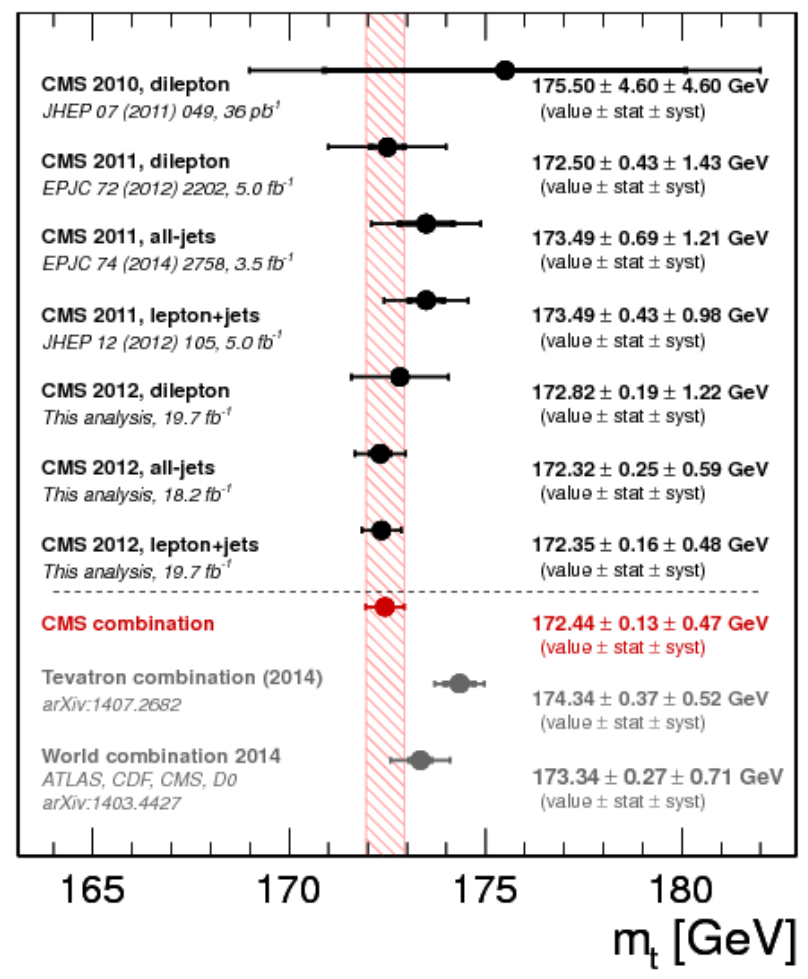

Figure 1: Summary of the "standard" measurements and their combination. The thick error bars show the statistical uncertainty and the thin error bars show the total uncertainty. Also shown are the 2014 Tevatron and world average combinations. 
These measurements are combined with previous measurements performed at $\sqrt{s}=7 \mathrm{TeV}$, shown in Fig. 1. The measurement performed in the lepton+jets channel at $\sqrt{s}=8 \mathrm{TeV}$, whose precision is unprecedented, carries a contribution of $72.5 \%$ in the combination, the latter resulting in a best linear unbiased estimate of $\mathrm{m}_{\mathrm{t}}=172.44 \pm 0.13$ (stat) \pm 0.47 (syst) GeV. The total uncertainty is dominated by the systematic uncertainty, whose main sources are the modeling of hadronization $(\sim 0.35 \mathrm{GeV})$, the JEC uncertainty $(\sim 0.15 \mathrm{GeV})$, and the modeling of the hard process - which includes renormalization and factorization scales, jet-to-parton matching, and matrix-element generator $-(\sim 0.15 \mathrm{GeV})$. As these sources are strongly correlated among the different measurements, the uncertainties are not reduced very much by combining the individual measurements. The total uncertainty is nevertheless below $0.3 \%$.

\section{2. "Alternative" methods}

One way to further improve the precision on $\mathrm{m}_{\mathrm{t}}$ could be to use large sample data to better constrain several tunable parameters in $t \bar{t}$ event modeling. For instance, pioneering studies have been already performed at $\sqrt{s}=8 \mathrm{TeV}$ for the modeling of fragmentation [2], color reconnection, and underlying event [3]. Another approach consists in designing "alternative" measurements, by considering alternative event topologies or observables that are sensitive to $\mathrm{m}_{\mathrm{t}}$ variations. While theoretically-calculable observables give the possibility to measure $\mathrm{m}_{\mathrm{t}}$ in a well defined renormalization scheme, other observables requiring only a partial reconstruction of the $t \bar{t}$ kinematics and other topologies present the advantages of alternative systematic sensitivity.

\subsection{Alternative event topologies}

The requirement of one forward (i.e. an absolute pseudorapidity $|\eta|>2.5$ ) light jet leads to a sample enriched in t-channel single top quark $(71 \%)$ rather than $t \bar{t}(<10 \%)$ events. The top quark

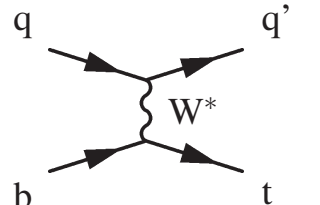

Figure 2: Dominant Feynman diagrams for single top quark production in the t channel. mass is inferred from the invariant mass of the top quark decay products, with the neutrino momentum being determined from $E_{\mathrm{T}}^{\text {miss }}$, through a calibration procedure. Applied to $8 \mathrm{TeV}$ data, this technique results in $\mathrm{m}_{\mathrm{t}}=172.60 \pm 0.77$ (stat) ${ }_{-0.93}^{+0.97}$ (syst) GeV [4]. The main sources of systematic uncertainty are JEC $(\sim 0.65 \mathrm{GeV})$, background calculations $(\sim 0.40 \mathrm{GeV})$, and fit calibration $(\sim$ $0.40 \mathrm{GeV})$. Since single top quark production, whose dominant Feynman diagram is represented in Fig. 2, is electroweakly mediated, parton distribution functions (PDF) as well as color reconnection and hard scattering modeling are different than in $\mathrm{t}_{\mathrm{t}}$ events, and the subsequent systematic uncertainties are thus weakly correlated.

\subsection{Theoretically-calculable observables}

A comparison of the measured tt production cross section to the expected one, e.g. computed with TOP++ at the next-to-next-to-leading order with next-to-next-to-leading-logarithm gluon resummation, gives access to the top quark pole mass $\left(\mathrm{m}_{\mathrm{t}}^{\text {pole }}\right)$. At $\sqrt{s}=7$ and $8 \mathrm{TeV}$, only dilepton 
$\mathrm{e}^{ \pm} \mu^{\mp}$ candidates are considered and the total uncertainty on the measured $\bar{t} \bar{t}$ cross section is dominated by the luminosity uncertainty [5]. At $\sqrt{s}=13 \mathrm{TeV}$, lepton+jets candidates are used and the systematic and luminosity uncertainties are of same order [6].

The top quark pole mass can also be extracted from differential distributions, such as the differential cross section with respect to $\rho_{S}=2 \times 170 \mathrm{GeV} / m(\mathrm{t} \overline{\mathrm{t}}+$ jet $)$, where $m(\mathrm{t} \overline{\mathrm{t}}+$ jet $)$ is the mass of the $t \bar{t}+$ jet system obtained after a kinematic reconstruction of dilepton t $\bar{t}$ events with additional hard jets $\left(p_{\mathrm{T}}>50 \mathrm{GeV}\right)$. Data recorded at $\sqrt{s}=8 \mathrm{TeV}$ are unfolded at particle level in the visible phase space with MADGRAPH+PYTHIA6 and compared to the next-to-leading-order cross section predicted with POWHEG+PyTHIA8 for several $\mathrm{m}_{\mathrm{t}}^{\text {pole }}$ values [7]. The highest sensitivity to $\mathrm{m}_{\mathrm{t}}^{\text {pole }}$ variations is observed for $\rho_{S} \sim 0.4$ and $\rho_{S}>0.6$. The theoretical modeling of the $\bar{t}+$ jet system is the main source of uncertainty.

These $\mathrm{m}_{\mathrm{t}}^{\text {pole }}$ measurements are presented in Fig. 3. Though not competitive in term of precision, they are compatible with the "standard" $\mathrm{m}_{\mathrm{t}}$ measurements that rely on simulation-based calibrations.

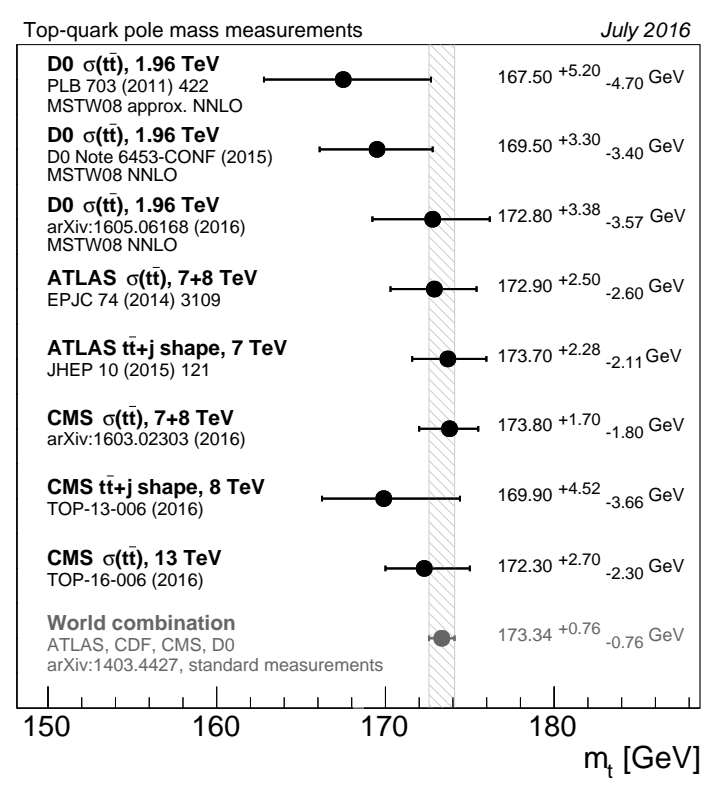

Figure 3: Summary of $\mathrm{m}_{\mathrm{t}}^{\text {pole }}$ measurements at Tevatron and LHC.

\subsection{Other observables}

Avoiding the full reconstruction of $\mathrm{b}$ jets gives the possibility to measure $\mathrm{m}_{\mathrm{t}}$ with a reduced sensitivity to the JEC uncertainty.

Rather than $b$ jets, secondary vertices typically left by $\mathrm{b}$ hadron decays can be used to infer $\mathrm{m}_{\mathrm{t}}$. Indeed, in dilepton and lepton+jets $\bar{t} \bar{t}$ events, the combination of the secondary vertex reconstructed from 3,4 , or 5 tracks within a jet and the isolated lepton $\ell$, associated to a $\rightarrow(\mathrm{W} \rightarrow \ell v) \mathrm{b}$ decay as shown in Fig. 4, has an invariant mass that is

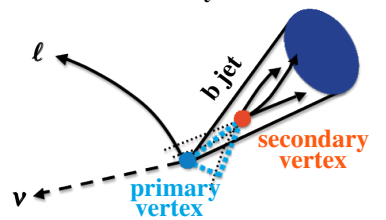

Figure 4: Final state products of a leptonic top quark decay. Tracks are represented by arrows. 
sensitive to $\mathrm{m}_{\mathrm{t}}$ variations. This technique, applied at $\sqrt{s}=8 \mathrm{TeV}$, results in $\mathrm{m}_{\mathrm{t}}=173.68 \pm$ 0.20 (stat) ${ }_{-0.97}^{+1.58}$ (syst) GeV [2]. The drawback of this method is the strong sensitivity to b quark hadronization $(\sim 1 \mathrm{GeV})$ and top quark $p_{\mathrm{T}}(\sim 0.8 \mathrm{GeV})$ modeling, mostly responsible for the systematic uncertainty.

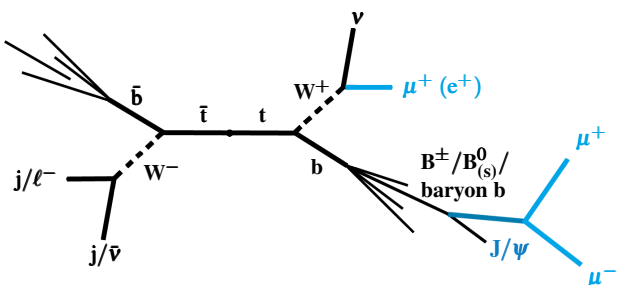

Figure 5: Pictorial view of an exclusive $\mathrm{J} / \psi$ production in a t⿱t system.

Instead of secondary vertices, only $\mathrm{J} / \psi \rightarrow$ $\mu^{+} \mu^{-}$decays can be considered. The corresponding topology is sketched in Fig. 5. The top quark mass is extracted through its correlation to the invariant mass of the $\mathrm{J} / \psi+\ell$ system. Experimentally, this decay channel has a very clean signature, but a very low branching fraction. At $\sqrt{s}=8 \mathrm{TeV}$, it leads to $\mathrm{m}_{\mathrm{t}}=173.5 \pm 3.0$ (stat) \pm 0.9 (syst) GeV [8]. Though statistically limited for now, this technique seems to be promising as the $\mathrm{b}$ quark hadronization uncertainty is only of $0.30 \mathrm{GeV}$ and the relevant experimental uncertainties are below $0.10 \mathrm{GeV}$.

With two identical decay branches ending in invisible particles, the dilepton channel presents a topological resemblance with some non-SM processes like the one shown in Fig. 6. A "stransverse mass" $\left(\mathrm{M}_{\mathrm{T} 2}^{\mathrm{bb}}\right)$ can thus be computed in SM t⿱㇒t events, treating $\mathrm{W}$ bosons as "child" particles and the only "upstream" mo-

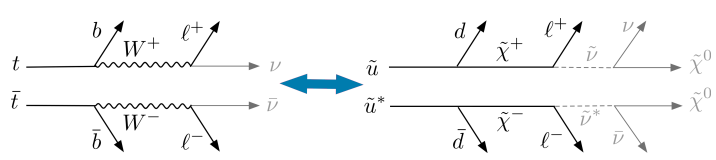

Figure 6: Similar signature of dilepton $t \bar{t}$ and $\tilde{u}^{*}{ }^{*}$ decays, where $\tilde{u}$ denotes the u-squark of the supersymmetry and $\tilde{u}^{*}$ its conjugate. mentum source being the initial state radiation. The distribution of $\mathrm{M}_{\mathrm{T} 2}^{\mathrm{bb}}$ is represented in Fig. 7a for several $m_{t}$ values. By construction, the highest sensitivity to $m_{t}$ variations is observed around the kinematic endpoint. The same phenomenon happens for the invariant mass of the $b$ jet plus isolated lepton combination $\left(\mathrm{M}_{\mathrm{b} \ell}\right)$ shown in Fig. $7 \mathrm{~b}$. An estimation of the $\mathrm{M}_{\mathrm{T} 2}^{\mathrm{bb}}$ and $\mathrm{M}_{\mathrm{b} \ell}$ distribution shapes from Gaussian Processes can be used either to measure $m_{t}$ alone or simultaneously with a JSF. The combination of both $m_{t}$ measurements, with a weight of 0.2 for the $m_{t}$ value obtained

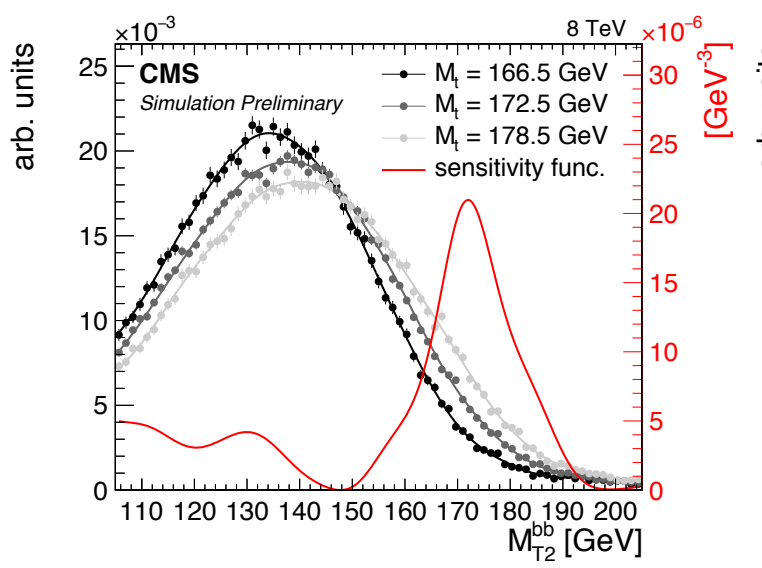

(a)

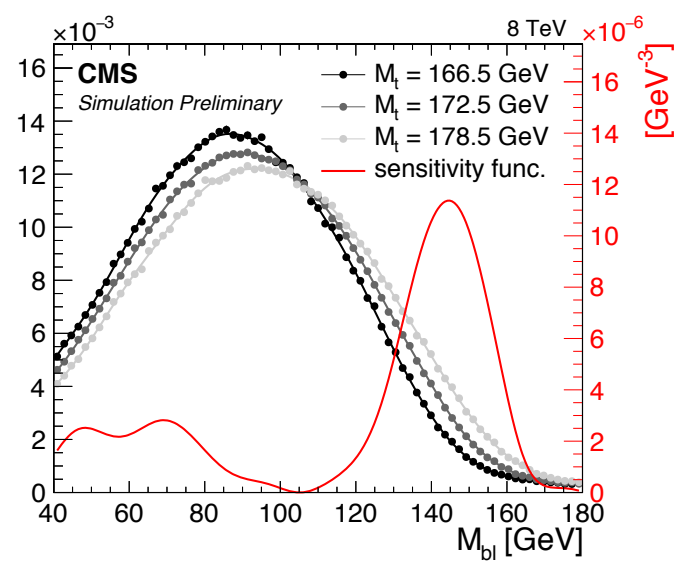

(b)

Figure 7: The $\mathrm{M}_{\mathrm{T} 2}^{\mathrm{bb}}$ (a) and $\mathrm{M}_{\mathrm{b} \ell}$ (b) distribution shapes in simulation corresponding to 3 values of $\mathrm{m}_{\mathrm{t}}$ are shown in gray [9]. The "local shape sensitivity" function is shown in red. 
with a simultaneous fit, leads to $\mathrm{m}_{\mathrm{t}}=172.22 \pm 0.18$ (stat) ${ }_{-0.93}^{+0.89}$ (syst) $\mathrm{GeV}$ [9]. The main sources of systematic uncertainty are similar to those of the "standard" measurement in the same decay channel but their value is smaller and this alternative measurement is eventually more precise.

\section{Conclusion and outlook}

"Standard" measurements result in a precision on $\mathrm{m}_{\mathrm{t}}$ better than $0.5 \mathrm{GeV}$. Nevertheless, the total uncertainty is dominated by the systematic uncertainty, which appears to be difficult to further reduce with these sole techniques. Several "alternative" measurements, involving either different topologies or observables and thus presenting an alternative systematic sensitivity, seem very promising.

"Standard" measurements, as well as most "alternative" ones, give access to $\mathrm{m}_{t}$ through simulation-based calibrations that need to be re-interpreted in view of a well-defined mass scheme. Progress towards this interpretation has been shown in this conference in Ref. [10]. A full consistency is yet observed between all $\mathrm{m}_{\mathrm{t}}$ and $\mathrm{m}_{\mathrm{t}}^{\text {pole }}$ measurements.

\section{References}

[1] CMS Collaboration, "Measurement of the top quark mass using proton-proton data at $\sqrt{s}=7$ and 8TeV", Phys. Rev.D 93 (2016) 2004, doi:10.1103/PhysRevD.93.072004, arXiv:1509.04044.

[2] CMS Collaboration, "Measurement of the top quark mass using charged particles in pp collisions at $\sqrt{s}=8 \mathrm{TeV} "$, Phys. Rev. D 93 (2016) 2006, doi:10.1103/PhysRevD.93.092006, arXiv:1603.06536.

[3] CMS Collaboration, "Study of the underlying event, b-quark fragmentation and hadronization properties in ț events", CMS Physics Analysis Summary CMS-PAS-TOP-13-007, CERN, 2013.

[4] CMS Collaboration, "Measurement of the top quark mass from single-top production events", CMS Physics Analysis Summary CMS-PAS-TOP-15-001, CERN, 2016.

[5] CMS Collaboration, "Measurement of the tt production cross section in the e $\mu$ channel in proton-proton collisions at $\sqrt{s}=7$ and $8 \mathrm{TeV} "$, JHEP $\mathbf{0 8}$ (2016) 029, doi:10.1007/JHEP08 (2016)029, arXiv:1603.02303.

[6] CMS Collaboration, "A measurement of the inclusive tt' production cross section in proton-proton collisions at $\sqrt{s}=13 \mathrm{TeV}$ using events with one isolated charged lepton and at least one jet", CMS Physics Analysis Summary CMS-PAS-TOP-16-006, CERN, 2016.

[7] CMS Collaboration, "Determination of the normalized invariant mass distribution of $\mathrm{t} t+\mathrm{jet}$ and extraction of the top quark mass", CMS Physics Analysis Summary CMS-PAS-TOP-13-006, CERN, 2016.

[8] CMS Collaboration, "Measurement of the mass of the top quark in decays with a $\mathrm{J} / \psi$ meson in $\mathrm{pp}$ collisions at 8TeV", (2016), arXiv:1608.03560. Submitted to JHEP.

[9] CMS Collaboration, "Measurement of the top quark mass in the dileptonic tt decay channel using the $\mathrm{M}_{\mathrm{b} \ell}, \mathrm{M}_{\mathrm{T} 2}$, and MAOS $\mathrm{M}_{\mathrm{b} \ell v}$ observables", CMS Physics Analysis Summary CMS-PAS-TOP-15-008, CERN, 2016.

[10] M. Preisser, "Top quark mass calibration for Monte-Carlo event generators", these proceedings. 